\title{
Impact of velocity gradient in Poiseuille flow on the statistics of coherent radiation scattered by flowing Brownian particles in optical coherence tomography
}

Ivan Popov

Andrew Weatherbee

Alex Vitkin 


\title{
Impact of velocity gradient in Poiseuille flow on the statistics of coherent radiation scattered by flowing Brownian particles in optical coherence tomography
}

\author{
Ivan Popov, ${ }^{\mathrm{a}, *}$ Andrew Weatherbee, ${ }^{\mathrm{a}}$ and Alex Vitkin ${ }^{\mathrm{a}, \mathrm{b}, \mathrm{c}}$ \\ ${ }^{a}$ University of Toronto, Department of Medical Biophysics, Toronto Medical Discovery Tower, Toronto, Ontario, Canada \\ bUniversity of Toronto, Department of Radiation Oncology, Toronto, Ontario, Canada \\ 'Ontario Cancer Institute/University Health Network, Division of Biophysics and Bioimaging, Toronto, Ontario, Canada
}

\begin{abstract}
A closed-form expression is obtained for the temporal correlation function of the scattered radiation detected in optical coherence tomography (OCT), taking into account the flow velocity gradient across the OCT detection volume in the suspension of flowing Brownian particles. The analytical approach we use includes both the laser beam and wavefront curvature radii changing over the depth. Also, we compare our results with a previously obtained theoretical model, partially an empirical approach. Our findings suggest the importance of the flow velocity gradient for accurate measurements of flow velocity vector, particle diffusivity, shear-induced diffusion, and potentially other OCT applications. (-) The Authors. Published by SPIE under a Creative Commons Attribution 4.0 Unported License. Distribution or reproduction of this work in whole or in part requires full attribution of the original publication, including its DOI. [DOI: 10.1117/1.JBO.24.9.097001]
\end{abstract}

Keywords: statistical optics; optical coherence tomography; scattering; blood flow; diffusion.

Paper 190026RR received Jan. 25, 2019; accepted for publication Aug. 23, 2019; published online Sep. 27, 2019.

\section{Introduction}

Optical coherence tomography (OCT) is an emerging modality most commonly used for 2-D and 3-D biotissue microstructural imaging. ${ }^{1}$ It also allows for additional contrast detection modes, for example, blood flow measurements. ${ }^{2}$ Further, OCT dynamic light scattering techniques have demonstrated simultaneous localized measurements of the velocity and diffusivity of flowing particles suspended in a liquid, enabling determination of both translational and Brownian motion parameters. ${ }^{3-6}$ This is potentially important in a biomedical context, for example, hemodynamically, where blood diffusivity carries important information about non-Newtonian properties of blood (e.g., shear-rate-dependent viscosity), which affects the blood flow; further, the shear-induced diffusion plays a crucial role in dynamics of the blood flow circulation. ${ }^{7}$ In addition, blood diffusivity contains potential information on blood glucose levels. ${ }^{8}$ Although accurately measuring and properly understanding the details of these diffusion and flow effects is difficult, it is biomedically desirable.

Blood diffusivity measurements also carry information about blood viscosity, whose increase has been linked to many major cardiovascular risk factors; viscosity may also be modulated by blood glucose levels. ${ }^{9}$ Although accurate viscosity measurements are possible in the absence of flow or in a perpendicular measurement geometry, ${ }^{10}$ in flowing vessels in many practical situations, this is complicated as the angle between OCT axis and blood flow direction will not be $90 \mathrm{deg}$ and/or maybe unknown. This generates an additional Doppler frequency spread arising from the flow velocity gradient across the OCT detection volume. The resultant Doppler broadening effect also complicates the accurate determination of the flow velocity vector and overall impacts the dynamics of scattered radiation.

*Address all correspondence to Ivan Popov, E-mail: ivanpopov@msn.com
It has been found that the velocity gradients affect the spectral width of OCT signal from the particles flowing in the small capillary. ${ }^{3}$ However, no analytical solution for correlation function of scattered radiation has been suggested; Weiss et al. ${ }^{3}$ used a numerical approach by taking into account only the longitudinal flow velocity gradient. An analytical model of this effect has recently been published. ${ }^{11}$ However, this model is based on an empirical approach with an unknown range of applicability. To obtain more accurate results, we are taking into account the changing radii of the beam and wavefront curvature over depth. Further, existing models describing the radiation scattered from flowing Brownian particles are based on the free-space geometry, ${ }^{3,11,12}$ whereas it is well known that speckle statistics in free space and in the imaging plane of the optical system geometries are different; ${ }^{13}$ a detailed discussion of this issue is given in Refs. 4 and 5. Overall, accurate simulations are important for proper data interpretation, especially at positions outside the beam waist.

In this paper, we thus extend our recently developed analytical model ${ }^{5,6}$ for the correlation function of OCT backscattered radiation, by using the approach based on a Green's function of the optical system and explicitly incorporating the flow velocity gradient effects. The model is validated by corroborating OCT experiments with flowing aqueous suspension of Brownian particles (polystyrene microspheres). For biomedical relevance, we also apply our model to the measurements of flow-induced blood diffusion recently reported in the literature. ${ }^{14}$

\section{Mathematical Model of Scattering in the Presence of a Flow Gradient}

Consider an OCT system with a Gaussian beam emerging from a single-mode optical fiber, shaped by the sample arm optics, and illuminating a collection of small scatterers (Brownian particles) suspended in a liquid and flowing through a glass 
capillary. It is assumed that the flow is laminar. Apart from bulk translational flow motion, the particles are experiencing random chaotic displacements due to Brownian motion. The particle displacement $\Delta r$ is thus the sum of two motions: $\Delta r=v \tau+\Delta r_{b}$, where $v$ is flow velocity, $\Delta r_{b}$ is a random displacement due to Brownian motion with mean square value of $\left\langle\Delta r_{b}^{2}\right\rangle=6 D_{d} \tau,{ }^{15}$ and $D_{d}$ is particle diffusivity. As seen, the translational laminar flow motion is deterministic, whereas Brownian motion is the random process. Our purpose is to find the resultant correlation function of backscattered radiation in the fiber end face plane.

The case of uniform flow over the detection volume (as determined by the OCT point spread function at $1 / e$ level) was considered in previous publications. ${ }^{4-6,15}$ Briefly, in most practical situations, the temporal correlation function $C_{s}$ was found to be $C_{s}(\tau)=C_{s b}(\tau) \cdot C_{s t}(\tau)$, where $C_{s b}(\tau)$ and $C_{s t}(\tau)$ are the correlation function contributions due to Brownian and translational motions, respectively. The correlation functions and their corresponding frequency-domain power spectra $W_{s b}(f)$ and $W_{s t}(f)$ are given by the following equations:

$$
\begin{gathered}
C_{s b}(\tau)=\exp \left(-\frac{\tau}{\tau_{b}}\right), \quad W_{s b}(f)=\left[1+\left(\frac{f}{\Delta f_{b}}\right)^{2}\right]^{-1} \\
\text { with } \Delta f_{b}=\frac{1}{2 \pi \tau_{b}} \\
C_{s t}(\tau)=\exp \left[i 2 \pi f_{D} \tau-\left(\frac{\tau}{\tau_{t}}\right)^{2}\right], \\
W_{s t}(f)=\exp \left[-\frac{\left(f-f_{D}\right)^{2}}{\Delta f_{t}^{2}}\right], \quad \text { with } \Delta f_{t}=\frac{1}{\pi \tau_{t}}
\end{gathered}
$$

where

$$
\tau_{b}=\left[(2 k)^{2} D_{d}\right]^{-1},
$$

$\tau_{t}=\left\{v^{2}\left[\left(\frac{\sin (\theta)}{w_{0}}\right)^{2}+\frac{1}{2}\left(\frac{\cos (\theta)}{l_{c} / 2}\right)^{2}\right]\right\}^{-1 / 2}$

are the correlation times due to Brownian and translational motion, respectively. $k$ is the wavenumber, $D_{d}$ is particle diffusivity, $w_{0}$ is the Gaussian beam waist radius, $l_{c}$ is coherence length of the source full length at $1 / e$ level of correlation function of the optical field, $\theta$ is the Doppler angle, $f_{D}$ is Doppler frequency, and $v$ is flow velocity. Note that correlation time $\tau_{t}$ does not depend on Gaussian beam radius $w(z)$ along optical axis $z$ but depends only on the Gaussian beam radius $w_{0}$ at the waist.

The physical reason for this is as follows. At the Gaussian beam waist, the wavefront is plane. Therefore, in perpendicular geometry, when the particle is crossing the beam, it crosses only the Gaussian amplitude profile. When the particle crosses the beam at some distance from the waist, it also crosses spherical Fresnel zones (because of wavefront sphericity outside of beam waist). It means that in reflection, we will get not only amplitude variations proportional to Gaussian beam amplitude but also phase variations. A simple calculation shows that the power spectrum of radiation reflected by a particle has the same spectral width, independent on beam waist position relative to particle, both for perpendicular and Doppler angle geometries.

Note that Eq. (1) can be generalized to include the rotational diffusion of particles having the shape of an oblate ellipsoid ${ }^{15}$ [which may be relevant to red blood cell (RBC) scattering in human hemodynamics]. This means that our results for flow velocity gradient impact can be extended to include this more general case. Another important factor that can influence diffusion is particle shape deformation. To the best of our knowledge, there is no general quantitative approach available to estimate the impact of particle deformability on its diffusivity. As a first order approximation, the impact of small shape changes can be estimated by varying the radii of major and minor axis of oblate ellipsoid, while keeping its volume constant.

To include the velocity gradient effects into this formalism, we follow the steps outlined in Ref. 6 but with the flow velocity $v$ in the Doppler term $\exp \left(i 2 \pi f_{D} \tau\right)=\exp \left[i 2 \pi \tau \frac{2 v_{z}(x, y, z)}{\lambda}\right]$ being spatially varying according to the Poiseuille flow profile in a pipe:

$$
\begin{aligned}
\mathbf{v} & =\left(v_{x}, v_{y}, v_{z}\right) \\
& =\left(v_{m} \sin \theta\left(1-\frac{r^{2}}{R^{2}}\right), 0, v_{m} \cos \theta\left(1-\frac{r^{2}}{R^{2}}\right)\right) .
\end{aligned}
$$

Here, velocity vector $\mathbf{v}=\left(v_{x}, v_{y}, v_{z}\right)$ is given in the co-ordinate system with its origin at the intersection of OCT axis and capillary centerline. The co-ordinate axis $y$ is chosen such that $v_{y}=0 ; r^{2}=(x \cos \theta+z \sin \theta)^{2}+y^{2}$ is the (squared) distance between the capillary axis and point $(x, y, z), v_{m}$ is flow velocity at the center of capillary, and $R$ is the capillary radius [see Figs. 1(a) and 1(b)].

The translational part of the correlation function can now be expressed as follows:

$$
\begin{aligned}
& C_{s t}\left(z_{1}, \tau\right)=\frac{\sqrt{\alpha(0) \beta(0) \zeta(0)}}{\sqrt{\alpha(\tau) \beta(\tau) \zeta(\tau)}} \\
& \exp \left[\frac{\beta(\tau) \delta(\tau)^{2}+\gamma(\tau) \delta(\tau) \varepsilon(\tau)+\alpha(\tau) \varepsilon(\tau)^{2}}{4 \alpha(\tau) \beta(\tau)-\gamma(\tau)^{2}}\right. \\
& \left.\quad-\frac{z_{1}^{2}+\left(z_{1}-v_{z} \tau\right)^{2}}{\left(l_{c} / 2\right)^{2}}-2 v_{x}^{2} \tau^{2}\left(\frac{1}{w^{2}}+\frac{i k}{2 \rho}\right)-2 i k v_{m} \tau \cos \theta\right],
\end{aligned}
$$

$\alpha\left(z_{1}, \tau\right)=\frac{4}{w^{2}}-\frac{2 i k v_{m} \tau \cos ^{3} \theta}{R^{2}}$,

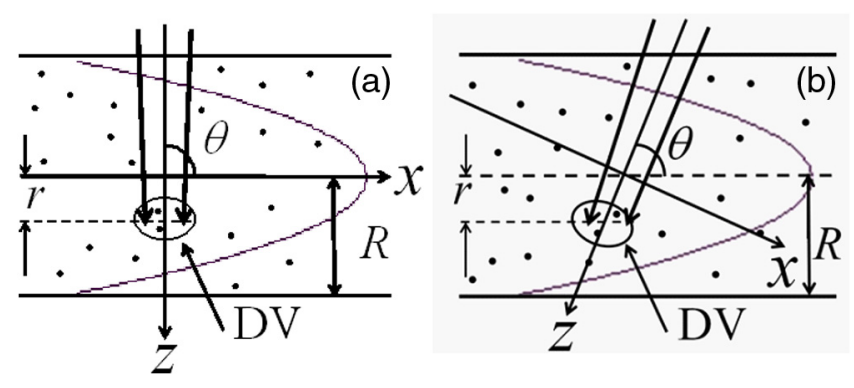

Fig. 1 Illumination geometry. (a) Perpendicular geometry, (b) slanted incidence geometry, $(x, z)$ is co-ordinate system, $y$ axis is perpendicular to $(x, z)$ plane, DV is the detection volume; parabolic-shaped curve shows Poiseuille flow velocity profile; the arrows show incident laser beam. 
$\beta\left(z_{1}, \tau\right)=\frac{2}{\left(\frac{l c}{2}\right)^{2}}-\frac{2 i k v_{m} \tau \cos \theta \sin ^{2} \theta}{R^{2}}$,

$\gamma\left(z_{1}, \tau\right)=\frac{2 i k v_{m} \tau \cos \theta \sin 2 \theta}{R^{2}}$

$\delta\left(z_{1}, \tau\right)=-4 v_{x} \tau\left(\frac{1}{w^{2}}+\frac{i k}{2 \rho}\right)$

$\varepsilon\left(z_{1}, \tau\right)=\frac{2\left(2 z_{1}-v_{z} \tau\right)}{\left(\frac{l c}{2}\right)^{2}}$

$\zeta\left(z_{1}, \tau\right)=\frac{4}{w^{2}}+\frac{2 i k v_{m} \tau \cos \theta}{R^{2}}$

In Eqs. (6)-(12), $w$ is the radius of the Gaussian beam at $1 / e^{2}$ intensity level, $\rho$ is the wavefront curvature radius, $z_{1}$ is the coordinate of the detection volume center along the OCT $z$ axis, and $v_{x}$ and $v_{z}$ are given by Eq. (5) with $r=z_{1} \sin (\theta) ; r$ values vary in the range between $-R$ and $+R$. The above analysis assumes the following: the calculations are performed in aberration-less approximation, only single scattering events are taken into account, and both $w$ and $l_{c} / 2$ are $\ll R$. These ranges are dictated by the fact that, in the real experimental situation, the tails of OCT point spread function are rather long and produce reflections from the capillary wall. These reflections in turn yield an interference pattern that distorts the laser beam profile and causes deviations not considered in our model. Further, the Poiseuille flow velocity profile is assumed as per Eq. (5). Note that Eq. (6) is valid only for the laser beam crossing the center of the capillary; the case of off-center is not considered here. Also, there are no limitations on the Doppler angle $\theta$, thus, the derived expressions should hold for any interrogation angle. Further, it is assumed that the scattering particles are small enough to produce the spherical wave in the far-field at the sample lens plane (i.e., we are assuming point-like scatterers) and are much smaller than the OCT detection volume.

The last term in the exponent of Eq. (6) is the Doppler shift corresponding to detection volume position at the capillary centerline. There are three other frequency shifting terms containing factor $i k v_{m} \tau$ in the expressions for $\alpha, \beta$, and $\gamma$. These four terms collectively yield the Doppler shift of the scattered radiation corresponding to spatially varying flow velocity [specifically $v_{z}(z)$ ]. Unfortunately, due to the complexity of analytical Eqs. (6)-(12), it is very difficult to assign a physical meaning to each term. We do note, however, that the terms containing capillary radius $R$ are caused by the flow velocity gradient. In the limit of $w / R \rightarrow 0, l_{c} / 2 R \rightarrow 0$ (i.e., in the limit of uniform flow velocity across the detection volume), we arrive at the result given by Eq. (2); thus, the correlation function $C_{s t}(\tau)$ converges to the expression obtained earlier for uniform flow conditions as expected. ${ }^{6}$ Let us also note that Eqs. (6)-(12) can be easily generalized to different beam and wavefront curvature radii in $x$ and $y$ directions. This situation may appear, for example, due to cylindrical lensing effect in the capillary wall. However, our calculations in (aberration-less) approximation show that in the experimental situations considered below, this effect does not influence the correlation function.
Let us analyze the factors $\sqrt{\alpha(0) / \alpha(\tau)}, \sqrt{\beta(0) / \beta(\tau)}$, and $\sqrt{\zeta(0) / \zeta(\tau)}$ that appear in front of the exponent in Eq. (6). The decay times at $1 / e$ level for $\alpha, \beta$, and $\zeta$ terms are as follows:

$$
\begin{aligned}
\tau_{\alpha} & =\sqrt{e^{4}-1} \lambda R^{2} /\left[\pi w^{2} v_{m} \cos ^{3} \theta\right], \\
\tau_{\beta} & =\sqrt{e^{4}-1} \lambda R^{2} /\left[\pi\left(\frac{l_{c}}{2}\right)^{2} v_{m} \cos \theta \sin ^{2} \theta\right], \\
\tau_{\zeta} & =\sqrt{e^{4}-1} \lambda R^{2} /\left[\pi w^{2} v_{m} \cos \theta\right] .
\end{aligned}
$$

The estimates of these decay times in experimental situations considered below show that they are much larger than those corresponding to the exponential term in Eq. (6) and thus can be safely neglected. Examining the square bracket term in the exponent of Eq. (6), it is impossible to derive a simple analytical expression for its decay time, so it will be estimated numerically for experimental situations considered below.

As mentioned in Sec. 1, a model of correlation function has recently been derived. ${ }^{11}$ To compare our more elaborate predictions of Eq. (6) with the results obtained in Ref. 11, we show the expression for translational part of correlation function, which follows from Eq. (20) in Ref. 11:

$C_{s t g}(\tau)=\exp \left[i 2 \pi f_{D} \tau-\left(\frac{\tau}{\tau_{t}}\right)^{2}-\left(\frac{\tau}{\tau_{\text {grad }}}\right)^{2}\right]$

Here, $\tau_{t}$ is given by Eq. (4), and

$\tau_{\text {grad }}=\left\{\frac{k v_{m} z_{1} \cos 2 \theta}{2 R^{2}} \sqrt{\left(w_{1}(z) \cos \theta\right)^{2}+\frac{1}{2}\left(\frac{l_{c}}{2} \sin \theta\right)^{2}}\right\}^{-\frac{1}{2}}$.

In Eqs. (16) and (17), we have used our notations and coordinate system, where $w_{1}(z)$ is the laser beam radius affected by the optical system aberrations. To evaluate it, the paraxial approximation is suggested. For our experimental setup, the paraxial approximation yields no change of the laser beam radius in the direction of $x$ axis. Therefore, we will set $w_{1}(z)=$ $w(z)$; this is introduced empirically in Ref. 11 as a defocus aberration.

The power spectrum corresponding to correlation function of Eq. (16) can be written as

$$
\begin{aligned}
W_{t g}(f) & =\exp \left[-\frac{\left(f-f_{D}\right)^{2}}{\Delta f_{t g}^{2}}\right], \\
\text { with } \Delta f_{t g} & =\frac{1}{\pi} \sqrt{\frac{1}{\tau_{t}^{2}}+\frac{1}{\tau_{\text {grad }}^{2}}} .
\end{aligned}
$$

Later on, we will use Eq. (18) to compare it with the spectrum given by our model.

It is also interesting to note that, in the perpendicular geometry $(\theta=90 \mathrm{deg})$, all three correlation functions given by Eqs. (2), (6), and (16) converge to the same expression. This can be proven by using the well-known equations for the Gaussian beam radius and wavefront curvature: ${ }^{16}$ 


$$
\begin{aligned}
& w=w(z)=\sqrt{1+\left(\frac{z-c}{z_{F}}\right)^{2}}, \\
& \rho=\rho(z)=z-c+\left(\frac{z_{F}}{z-c}\right)^{2},
\end{aligned}
$$

where $z_{F}=k w_{0}^{2} / 2$ is the Rayleigh range of the Gaussian beam, and $c$ is the position of the beam waist. It is thus only with departure from perpendicularity that important differences arise, as shown below.

Summarizing the theoretical derivations, we present a closed-form analytical expression for the correlation function of coherent radiation scattered by flowing Brownian scatterers exhibiting a flow velocity gradient. Unlike the mathematical model in Ref. 11 based partially on the empirical approach, our model of the correlation function is based on the rigorous analytical analysis with well-defined applicability range. We now move on to the experimental phantom validations and a preclinical application example of our derived formulations.

\section{Experimental Setup}

For experimental validation, a research-grade fiber-based spectral domain OCT system was used, operating in M-mode for this study. ${ }^{10}$ Signals recorded at the photodetector are processed into A-scans, which represent the depth-resolved reflectivity profiles of the sample at a fixed lateral position. M-mode operation was obtained by choosing the signals from the fixed depth of consecutive A-scans (thus as a function of time).

In the sample arm, the Gaussian beam emitted from the singlemode fiber and shaped by an optical system had $w_{0}=11.5 \mu \mathrm{m}$ at $1 / e^{2}$ intensity level in air (depth of field $\left.800 \mu \mathrm{m}\right)$. The axial OCT coherence length measured $l_{c}=13 \mu \mathrm{m}$ (full width at $1 / e$ signal level (here signal represents the Fourier transform of spectrometer output) in air. A microbore glass capillary with an inner diameter of $165 \mu \mathrm{m}$ was placed perpendicularly or at the angle $\theta=$ $82.3 \mathrm{deg}$ to the OCT optical axis in the sample arm. The sample beam was focused $700 \mu \mathrm{m}$ below the center of the capillary, to ensure varying beam size across it (23 to $27 \mu \mathrm{m}$ range).

The scattering phantom consisted of 215-nm-diameter monodispersed polystyrene microspheres suspended in water. The small microsphere size was selected to satisfy the model assumptions of scatterers being much smaller than the detection volume. The microsphere concentration was $0.5 \%$, resulting in scattering coefficient (calculated via Mie theory) of $0.22 \mathrm{~cm}^{-1}$; the relatively low concentration of particles was chosen to ensure the single scattering regime. The flow was controlled by a syringe pump, with the flow velocity $v_{m}=1.94 \mathrm{~mm} / \mathrm{s}$ at the capillary center, as calculated from the measured mass discharge.

\section{Results and Discussion}

Since the correlation function in Eq. (6) exhibits a rather complicated dependence on time, we used numerical Fourier transform to obtain its power spectrum. Numerical calculations of the resulting power spectra yield nearly Gaussian functions (to within 1\%) for the parameters in the experimental situation described above. Similarly, the correlation function given by Eq. (6) in perpendicular geometry and the envelope of it in the Doppler angle geometry are similarly close to Gaussian functions. This implies that the experimentally obtained spectrum is well described by the Voigt function. ${ }^{4}$ We thus fitted the experimentally obtained power spectra to a Voigt function by using a two-parameter fit, simultaneously obtaining the Brownian and translational correlation times $\tau_{b}$ and $\tau_{t}$. The spectral approach is widely used in the literature with one of the reasons being that it's convenient in the interpretation of results obtained: the wider the spectral width, the larger the velocity in flow measurement experiments.

Figure 1 illustrates the measurement geometries and the co-ordinate systems pertinent to the analysis of the scattering model. In this figure, $z$ is the direction of OCT optical axis, DV is the detection volume, and the parabolic-shaped curve shows Poiseuille velocity profile.

Figure 2 shows the correlation functions (a) and power spectra (b) obtained from the raw experimental data for perpendicular and Doppler angle geometries, at a distance $r=$ $40 \mu \mathrm{m}$ from capillary center. Also shown are the theoretical correlation functions given by $C_{s}(\tau)=C_{s b}(\tau) \cdot C_{s t}(\tau)$, where $C_{s b}(\tau)$ and $C_{s t}(\tau)$ are given by Eqs. (1) and (6) correspondingly. In these equations, the following parameters of experimental setup were used: $w=24 \mu \mathrm{m}, \rho=0.987 \mathrm{~mm}, \theta=90 \mathrm{deg}$ for perpendicular geometry, $\theta=82.3 \mathrm{deg}$ for Doppler angle geometry. The correlation time due to Brownian motion was found in the no-flow experiment to be $\tau_{b}=2.80 \mathrm{~ms}$. The theoretical power spectra were calculated by using numerical Fourier transform of correlation functions. As seen, the agreement between theoretical and experimental dependencies is good.

As already explained, by using the experimental spectra fitted to Voigt function, we were able to recover correlation time $\tau_{t}$ due to translation. From the latter, the experimental plot of the half spectral width at $1 / e$ level $\Delta f_{t}=1 /\left(\pi \tau_{t}\right)$ as a function of axial co-ordinate $z$ was constructed. The details of Voigt function fitting to experimental data are given in Ref. 10. Further, the particle diffusivity values were obtained from $D_{d}=\left[(2 k)^{2} \tau_{b}\right]^{-1}$ using both perpendicular and oblique incidence geometries (see Fig. 1), as the Brownian motion parameters should be independent of the interrogation angle. Indeed, the two recovered values of diffusivity at $z=40 \mu \mathrm{m}$ were within $<0.4 \%$ of each other, suggesting that the small departure of the power spectra from Gaussian profile is insignificant.

Figure 3(a) shows the spectral widths obtained from Eqs. (2) and (4) and the experimental results as a function of the distance between the detection volume and capillary axis $r=z \cdot \sin \theta$ for Doppler angle $\theta=90 \mathrm{deg}$. Although the laser beam is converging and its radius is thus varying, this does not affect the symmetrical shape of dependence $\Delta f_{t}(z)$ for this perpendicular interrogation geometry: the spectral width depends on laser
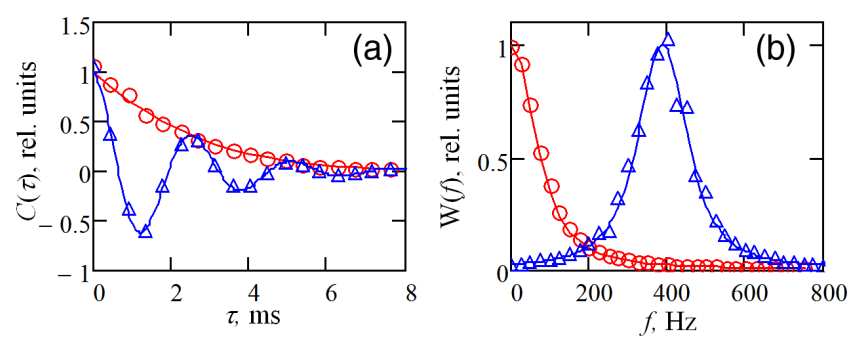

Fig. 2 (a) Correlation functions and (b) power spectra of scattered radiation at $r=40 \mu \mathrm{m}$. (a) Red circles: experimental data for perpendicular geometry, blue triangles: Doppler angle geometry; solid lines: calculations based on the product of correlation functions in Eqs. (1) and (6). (b) Red circles: experimental data for perpendicular geometry, blue triangles: Doppler angle geometry, solid lines: calculations based on the Fourier transform of correlation functions shown in (a). 

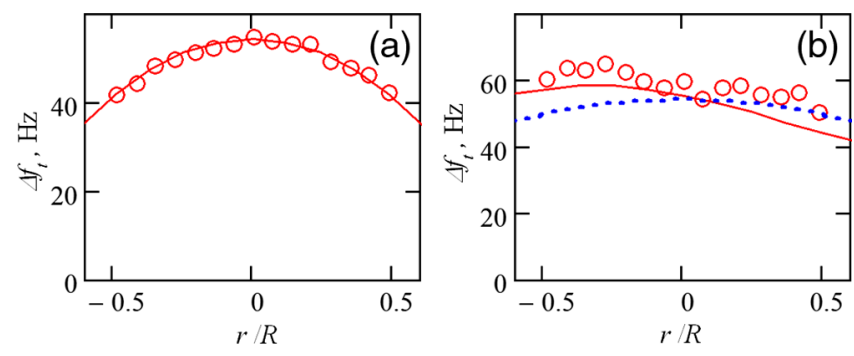

Fig. 3 Spectral width $\Delta f_{t}$ as a function of relative displacement of the detection volume from capillary center $r / R$. (a) Theory (solid line) and experimental data (circles) for Doppler angle $\theta=90 \mathrm{deg}$; the calculations are based on Eqs. (2) and (4) obtained under uniform flow assumption [those based on Eqs. (6) and (18) yield identical results]. (b) Theory (solid line) based on Fourier transform of Eq. (6), calculations based on Eq. (18) ${ }^{11}$ (blue dotted line), and experimental results (circles) for Doppler angle $\theta=82.3 \mathrm{deg}$.

beam radius at the waist only. As seen from Fig. 3(a), the agreement between the theoretical predictions and experimentally obtained spectral widths is very good. As previously mentioned, at this perpendicular geometry, all three models: our previous simpler formulation of Eq. (2), our current gradient-including result [based on Fourier transform of Eq. (6)], and the published literature model via Eq. (18) predict identical behavior. We also note that according to Eqs. (2) and (4), the spectral width has the same dependence on displacement $r$ as flow velocity profile $v(r)$. And the parabolic shape of the spectral width seen in Fig. 3(a) (see below), with its maximum at the centerline, suggests that indeed $v(r)$ exhibits a Poiseuille velocity profile, i.e., given by Eq. (5). This laminar regime is also consistent with the low Reynolds number: $\operatorname{Re}=\rho v l / \eta=0.36$ (water density $\rho=1 \mathrm{~g} / \mathrm{cm}^{3}$, flow velocity $v=2 \mathrm{~mm} / \mathrm{s}$, capillary diameter $l=165 \mu \mathrm{m}$, water viscosity $\eta=0.89 \mathrm{mPa} \cdot \mathrm{s}$ ).

Figure 3(b) shows the calculations of spectral width based on Fourier transform of Eq. (6), spectral width given by Eq. (18), and experimental results for the oblique geometry case of Doppler angle $\theta=82.3 \mathrm{deg}$. The results are shown in the interval $r / R<0.6$; at positions of detection volume near the wall, the wings of the Gaussian beam begin to show artefactual signals from the capillary wall reflections (and the wings of the negative frequency Doppler peak start leaking into the positive frequencies, deviating the measured spectral density from the Voigt profile). As seen, the dependence of $\Delta f_{t}$ on position of detection volume $r=z \cdot \sin \theta$ is rather different from the symmetric trend shown in Fig. 3(a): the maximum frequency shift is now no longer at the capillary center. The magnitude of the resultant spatial asymmetry increases with Doppler angle $\theta$ 's departure from $90 \mathrm{deg}$ (simulation results not shown); it is thus noticeable but not too extreme in comparing Figs. 3(a) and 3(b) for the selected $7.7 \mathrm{deg}$ deviation from perpendicularity. As seen in Fig. 3(b), our theoretical model of Eq. (6) agrees well (to within $<5 \%$ ) with experimental data results and certainly recapitulates the asymmetric trend in the data; conversely, the theoretical prediction of the literature model via Eq. (18) shows symmetrical behavior not supported by the data. Note that, although Eqs. (17) and (18) predict larger spectral width for larger laser spot radius, the contribution of the term containing laser beam radius $w\left(z_{1}\right)$ in Eq. (17) is very small in the near perpendicular geometry. Thus, the theoretical model in Ref. 11 fails to explain the asymmetrical trend of spectral width as a function of displacement $r$ in our experiment. A small departure of the experiment from the theory of Eq. (6) can be explained by the optical aberrations caused by slant incidence of laser beam on the glass capillary: the inner and outer walls of the capillary form a cylindrical lens, and with nonperpendicular incidence, this effect produces aberrations that broaden the laser spot inside the capillary, yielding a larger Doppler frequency spread across the detection volume.

This asymmetric trend in the nonperpendicular geometry is caused by scatterers in different positions within the detection volume producing different Doppler shifts, which contribute to the broadening of Doppler signal. The larger the beam spot radius $w(z)$, the larger this Doppler shift spread. Since the Gaussian beam is converging toward $z=700 \mu \mathrm{m}$ in our measured and simulated arrangements, the contribution to the spectral width of backscattered radiation due to the Doppler shift spread is larger in the negative $z$ range.

Let us now compare the predictions of our theoretical results and those published in Ref. 11 with experimental data for shearinduced diffusion in blood recently reported in the literature. ${ }^{14}$ A possible concern of our model's applicability and accuracy is whether multiple scattering contributes to the measured spectrum of scattered radiation. According to experimental results in Ref. 14, the recovered diffusivity shown in Fig. $2 \mathrm{C}$ of that publication is symmetric with respect to the venule center, implying that correlation time and spectral width are also symmetric with respect to the venule center. As is well known, multiple scattering broadens the spectral width, ${ }^{17}$ which would cause differential broadening across the vessel; thus, the detected symmetry implies that multiple scattering effects do not contribute significantly to the spectral width data. Further, in blood vessels of $\sim 50 \mu \mathrm{m}$ diameter, the velocity profile is parabolic within the study's measurement uncertainty, ${ }^{14}$ further suggesting that our model is directly applicable in this context.

A number of various size venules have been investigated in the reported preclinical study $;{ }^{14}$ here, we analyze the data from the second panel of Fig. $2 \mathrm{C},{ }^{14}$ with venule radius $R=25 \mu \mathrm{m}$; the measured flow velocity profile is reported in Ref. $14 v(r)=$ $v_{m}\left[1-(a r / R)^{2}\right]$ with $a=0.71$. The departure of $a$ from unity ( $a=1$ as per Poiseuille flow profile) is the manifestation of the fact that RBCs at the venule wall do not stick to it but continue moving. The other experimental parameters have the following values: Doppler angle $\theta=5 \mathrm{deg}$ (OCT axis nearly parallel to selected venule), laser wavelength $\lambda=1.31 \mu \mathrm{m}$ in air, laser beam waist radius $w_{o}=3.5 \mu \mathrm{m}$, coherence length $l_{c}=7 \mu \mathrm{m}$, and blood flow velocity at the venule center $v_{m}=7.5 \mathrm{~mm} / \mathrm{s}$.

Since the derived correlation function of scattered radiation now depends on the flow velocity gradient, we get a more complete physical description than the one based on the model given in Ref. 14, which is close to the one given by Eqs. (1) and (2). We will now apply this developed formalism to the experimental conditions in Ref. 14 summarized above. Our intention is to compare our model predictions and those derived from the model in Ref. 11 by applying them both to the following situations: (a) the interrogated depth is at the laser beam waist and (b) laser beam waist is shifted $40 \mu \mathrm{m}$ away, along $z$ axis. Note that only first (a) situation is considered in Ref. 14.

Calculations of the corresponding spectral widths obtained from the numerical Fourier transform of Eq. (6) are shown in Fig. 4(a). In this figure, we show the results of the B-scan simulation. Here, B-scan simulation calculates the spectral width as a function of lateral location, Doppler angle $\theta=5 \mathrm{deg}$, i.e., OCT axis nearly parallel to selected venule as is in the experiment in Ref. 14. Further, we are choosing only two depths, 

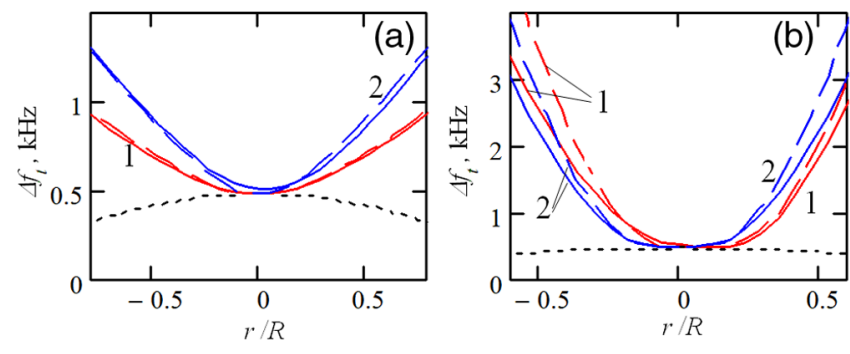

Fig. 4 Spectral width $\Delta f_{t}$ as a function of relative displacement from venule center $r / R$ at a Doppler angle $\theta=5 \mathrm{deg}$, corresponding to experimental conditions given in Fig. $2 \mathrm{C}$ in Ref. 14. (a) Simulations of B-scan experiment based on Fourier transform of Eq. (6), where we are analyzing the depth of the detection volume at the beam waist [line (1)] and $40 \mu \mathrm{m}$ away from the beam waist [line (2)]. Predictions of theory in Ref. 11 [Eq. (18) of this paper] are shown by the dashed lines. Dotted line is the spectral width given by Eq. (2) that assumes uniform flow velocity across the OCT detection volume. (b) Corresponding simulated A-scan experiment. In analogy with (a), solid lines are calculations based on Fourier transform of Eq. (6) with the position of the beam waist at the venule center [line (1)] and $40 \mu \mathrm{m}$ away along OCT $z$ axis [line (2)]; dotted line shows the spectral width given by Eq. (2); also shown by the dashed lines are the prediction of the theory given in Ref. 11 [Eq. (18)].

where the detection volume is either exactly at the beam waist or at a certain distance from it. It is also assumed that B-scan has been performed multiple times, so M-mode experiment data are available at each spatial point. In general, B-scan is the 2-D $(x, z)$ image frame obtained from many adjacent A-scans by laser beam scanning along the $x$ axis. A-scan is a single-line optical reflectivity profile as a function of depth along the OCT optical axis $z .^{18}$

The solid lines correspond to Gaussian beam radius of $w_{0}=$ $3.5 \mu \mathrm{m}$ (line (1): detection volume at the Gaussian beam waist as is in Ref. 14, experimental situation corresponds to that of Fig. 2C (right panel) and $w=5 \mu \mathrm{m}$ [line (2): detection volume $40 \mu \mathrm{m}$ away from beam waist along OCT $z$ axis). Dashed lines show corresponding data for spectral width given by the model in Ref. 11 [Eq. (18) of this paper]. We see that the difference between our model and that given in Ref. 11 is not that big here. The dotted line shows the spectral width given by Eq. (2), which corresponds to uniform flow that neglects velocity gradients [beam radius of $3.5 \mu \mathrm{m}$, for direct comparison with line (1)]. The differences between the two solid lines and the dotted curve behavior illustrate the importance of the flow velocity gradient impact on scattered radiation dynamics.

Figure 4(b) shows the spectral width as a function of $r$ in the simulated A-scan experiment for the same venule and same Doppler angle of $\theta=5 \mathrm{deg}$. Here, both the laser spot and wavefront curvature radii are changing significantly along $z$ axis [and as a function of $r=z \cdot \sin (\theta)]$. Here, we also see that the predictions of our model [solid lines (1) and (2)] are having the same trend as the theory given in Ref. 11 [dashed line, Eq. (18)]. The dotted line shows the spectral width for uniform flow motion given by Eq. (2). Again, the range of displacements shown in Fig. 4 is limited to the interval $r / R \leq 0.6$, as otherwise the laser spot begins to interrogate the venule wall and the theory does not account for this. Also note that these situations are not considered in Ref. 14.

Since the impact of flow velocity gradient is fully neglected in Ref. 14, the fitting procedure, which decouples translational and Brownian motions, has no room for the gradient term. In this situation, the fitting procedure will compensate the

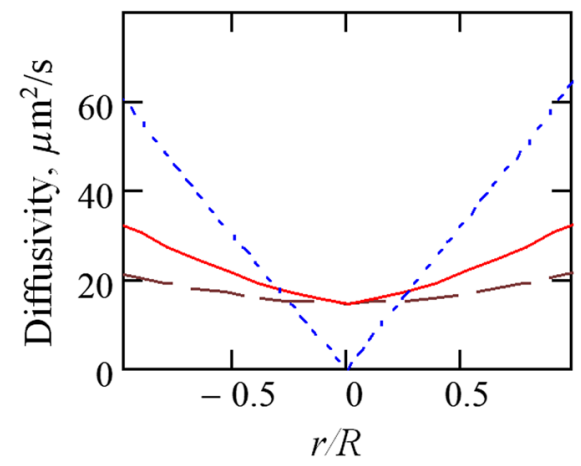

Fig. 5 Diffusivity as a function of displacement $r / R$. Solid and dashed lines are diffusivities $D_{d e}$ equivalent to spectral widths shown by solid lines (1) and (2) correspondingly in Fig. 4(a), as calculated from Eq. (20); dotted line is the measured diffusivity from Ref. 14 [Fig. 2C, right panel].

underestimated translational motion effects by increasing the value of the Brownian term. We thus expect that the shearinduced diffusivity found in Ref. 14 is overestimated.

To be able to compare the impact of shear-induced diffusion and that of flow velocity gradient on the scattered radiation spectra in the OCT experimental conditions of Ref. 14, we equate the spectral widths (at $1 / e$ level) due to diffusion $\Delta f_{b 1}=\sqrt{e-1}(2 k)^{2} D_{d} / 2 \pi$, which follows from Eq. (1), and that due to the velocity gradient $\Delta f_{t}$, obtained from the Fourier transform of Eq. (6). We get the following equation for equivalent diffusivity:

$D_{d e}=2 \pi \Delta f_{t} /\left[(2 k)^{2} \sqrt{e-1}\right]$.

Figure 5 shows the equivalent diffusion coefficients $D_{d e}$ as a function of relative displacement $r / R$ in the B-scan experiment, where only two depths are analyzed: dashed and solid lines correspond to the depths at the beam waist and $40 \mu \mathrm{m}$ away from it. Also shown by the dotted line is the shear-induced diffusion coefficient reported in the original article ${ }^{14}$ [Fig. 2C, second panel]. One can see from Fig. 5 that the range of diffusivities equivalent to spectral broadening caused by flow velocity gradient [solid and dashed lines] and that of flow-induced diffusion (dotted curve) is of the same order. It is thus important to account for the impact of flow velocity gradient on statistics of scattered radiation; this applies not only in this particular example of OCT determination of shear-induced diffusion but also in many other OCT experiments involving blood flow. The effect of Doppler broadening in the gradient flow should be also taken into account in the laser Doppler OCT flowmetry, ${ }^{2}$ and in particular, for transverse velocity component measurements of blood flow.

\section{Conclusions}

In summary, we have developed a closed-form analytical model for the OCT correlation function of coherent radiation scattered from a suspension of flowing Brownian particles, with explicit account for the flow velocity gradient and parameters of the Gaussian beam (radii of the beam and wavefront curvature). The validation experiments show good agreement between our derived model predictions and measurement results. It is also shown that the translational part of the correlation function and thus the power spectrum departs to a certain extent from the 
results based on the model, ${ }^{11}$ indeed, the formalism presented in Ref. 11 cannot explain correctly the trends in the dependence of spectral width as a function of detection volume displacement across the capillary in our experiments. We thus show that this effect should be included in the existing models of coherent radiation scattering in the Brownian particles and blood flows, ${ }^{3,11,12}$ which allow simultaneous measurements of flow velocity vector and RBC diffusivity. We further demonstrate the importance of including the flow velocity gradients in the recently reported measurements of shear-induced diffusion in blood vessels $;{ }^{14}$ in fact, we estimate its effect on the power spectrum to be of the same order of magnitude as the actual reported shearinduced diffusion.

\section{Disclosures}

The authors have no relevant financial interests in this article and no potential conflicts of interest to disclose.

\section{Acknowledgments}

This study was supported by the Canadian Institutes of Health Research (Grant No. 126172).

\section{References}

1. F. Fercher et al., "Optical coherence tomography-principles and applications," Rep. Prog. Phys. 66, 239-303 (2003).

2. V. X. D. Yang and I. A. Vitkin, "Principles of Doppler OCT," in Optical Coherence Tomography for Cardiovascular Research, E. Regar, T. G. van Leeuwen, and P. W. Serruys, Eds., pp. 305-319, Informa Healthcare, London (2007).

3. N. Weiss, T. G. van Leeuwen, and J. Kalkman, "Localized measurement of longitudinal and transverse flow velocities in colloidal suspensions using optical coherence tomography," Phys. Rev. E. 88, 042312 (2013).

4. I. Popov, A. S. Weatherbee, and I. A. Vitkin, "Dynamic light scattering arising from flowing Brownian particles: analytical model in optical coherence tomography conditions," J. Biomed. Opt. 19, 127004 (2014).

5. I. Popov and I. A. Vitkin, "Dynamic light scattering by flowing Brownian particles measured with optical coherence tomography: impact of the optical system," J. Biomed. Opt. 21, 017002 (2016).

6. I. Popov, A. S. Weatherbee, and I. A. Vitkin, "Statistical properties of dynamic speckles from flowing Brownian scatterers in the vicinity of the image plane in optical coherence tomography," Biomed. Opt. Express 8, 2004-2017 (2017).
7. T. Omori et al., "Shear-induced diffusion of red blood cells in a semidilute suspension," J. Fluid Mech. 724, 154-174 (2013).

8. H. Ullah et al., "Can temporal analysis of optical coherence tomography statistics report on dextrorotatory-glucose levels in blood?" Laser Phys. 21(11), 1962-1971 (2011).

9. G. Sloop, "A unifying theory of atherogenesis," Med. Hypotheses 47, 321-325 (1996).

10. A. Weatherbee, I. Popov, and I. A. Vitkin, "Accurate viscosity measurements of flowing aqueous glucose solutions with suspended scatterers using a dynamic light scattering approach with optical coherence tomography," J. Biomed. Opt. 22, 087003 (2017).

11. N. Uribe-Patarroyo and B. E. Bouma, "Velocity gradients in spatiallyresolved laser Doppler flowmetry and dynamic light scattering with confocal and coherence gating," Phys. Rev. E. 94, 022604 (2016).

12. J. Lee et al., "Dynamic light scattering in optical coherence tomography," Opt. Express 20(20), 22262-22277 (2012).

13. T. Yoshimura, "Statistical properties of dynamic speckles," J. Opt. Soc. Am. A. 3, 1032-1054 (1986).

14. J. Tang et al., "Shear-induced diffusion of red blood cells measured with dynamic light scattering-optical coherence tomography," $J$. Biophotonics 11, e201700070 (2017).

15. B. J. Berne and R. Pecora, Dynamic Light Scattering, Dover Publications, New York (2000).

16. A. Yariv, Quantum Electronics, p. 676, Wiley, New York, (1989).

17. K. K. Bizheva, A. M. Siegel, and D. A. Boas, "The transition to diffusing wave spectroscopy," Phys. Rev. E. 58(6), 7664-7667 (1998).

18. A. Gh. Podoleanu, "Optical coherence tomography," J. Microsc. 247(3), 209-219 (2012).

Ivan Popov received his master's degree in radiophysics and PhD in optics from St. Petersburg State University, Russia, in 1972 and 1979 , respectively, and earned his DSc degree in optics from the Institute of Fine Mechanics and Optics, St. Petersburg, Russia, in 2000. His area of expertise includes optics of speckles, non-contact techniques of motion measurement and biomedical applications of optical coherence-domain techniques. He is currently with the Biophotonics Group at the University Health Network, Toronto, Canada.

Andrew Weatherbee: Biography is not available.

Alex Vitkin is a professor of medical biophysics and radiation oncology at the University of Toronto, a senior scientist at the University Health Network, and a clinical medical physicist at Princess Margaret Cancer Centre (all in Toronto, Canada). He has published $>180$ papers and book chapters on biophotonics, primarily on tissue polarimetry and optical coherence tomography. He is also a fellow of OSA and of SPIE, and a professor at the Nizhny Novgorod State Medical Academy, Russia. 